\title{
Modeling the near-field of extremely large aperture arrays in massive MIMO systems
}

\author{
Botond Tamás Csathó, Bálint Péter Horváth and Péter Horváth
}

\begin{abstract}
Massive multiple-input multiple-output (MIMO) is a key technology in modern cellular wireless communication systems to attain a very high system throughput in a dynamic multi-user environment. Massive MIMO relies on deploying base stations equipped with a large number of antenna elements. One possible way to deploy base stations equipped with hundreds or thousands of antennas is creating extremely large aperture arrays. In this paper, we investigate channel modeling aspects of massive MIMO systems with large aperture arrays, in which many users are located in the near-field of the aperture. Oneand two-dimensional antenna geometries, different propagation models, and antenna element patterns are compared in terms of inter-user correlation, condition number of the multi-user channel matrix, and spectral efficiency to identify key design parameters and essential modeling assumptions. As our analysis reveals by choosing spectral-efficiency as a design objective, the size of the aperture is the critical design parameter.
\end{abstract}

Index Terms-massive MIMO, extremely large aperture arrays, near-field, channel modeling

\section{INTRODUCTION}

Massive multiple-input multiple-output (MIMO) is a state of the art physical layer technology, which is of key importance in modern wireless communication, moreover, an ingredient of 5G. In a massive MIMO system, the base station serves the mobile stations (MS) in the same time-frequency resource, while users are separated in the spatial domain. The core concept of massive MIMO is to have at least an order of magnitude higher number of antennas at the base station than the number of simultaneously transmitting mobile station antennas [1]. A mobile station can have an arbitrary number of antennas, although the number of antennas is limited due to the physical constraints of a handheld device. The large number of antennas at the BS will result in a significant number of different channels between an MS and the BS, which brings the benefits of the technology. It has been shown in [2], that by utilizing more antennas, the spectral efficiency (SE) will always increase. Therefore, the development of base stations with hundreds or thousands of antennas is expected. Interestingly, the increasing SE holds even with measured channel state information (CSI), linear precoding, and decoding [1].

The work of Björnson [3] considers extremely large aperture arrays (ELAA) [4] as a promising concept of massive MIMO. ELAA is one way of increasing the number of antennas, thus increasing the spectral efficiency. An essential property of the large aperture that its radiating near-field can stretch multiple kilometers. Therefore, some users are located in a region where the radiated electromagnetic (EM) waves cannot be

The authors are with the Budapest University of Technology and Economics Department of Broadband Infocommunications and Electromagnetic Theory. Email: bcsatho@evt.bme.hu, horvath.balint@hvt.bme.hu,hp@hvt.bme.hu approximated by plane-waves as in the far-field. Consequently, classical lobe-based channel models do not hold. Investigation of practical channel models, as well as the acquisition of the channel features in a particular deployment scenario are of current research interest [3].

There are existing works about the properties of large apertures, for example [5], [6] and [7]. Nevertheless, the authors in these papers focus on one-dimensional antenna arrays, with antenna element spacing less than two wavelengths. Although these assumptions are common in the literature, if an ELAA system will be built, it is likely to be two-dimensional. Furthermore, if considering frequencies higher than $1 \mathrm{GHz}$, the spacing between the antenna elements can easily be orders of magnitude larger than the wavelength. As a demonstrative example, one can consider an office block, where antenna elements are affixed to the windows of the building, resulting in inter-element spacing in the order of a few meters. In [5] and [6], line of sight (LoS) propagation is assumed. On the contrary, in a realistic scenario, there is at least one significant reflection from the ground, as it is assumed in [7].

Our work will focus on the effect of modeling accuracy in the near-field of an ELAA. One- and two-dimensional arrays will be compared, and the effect of ground reflection will also be taken into account. Inter-element spacing is considered to be an order of magnitude larger than the wavelength. Consequently, mutual coupling between antenna elements can be neglected. Furthermore, different models of each antenna element and different array geometries will be compared. The metrics of the analysis are the inter-user correlation (IUC), condition number $(\mathrm{CN})$ of the multi-user channel matrix, and spectral efficiency. The first goal is to analyze the effect of modeling accuracy of a realistic ELAA scenario. Furthermore, we aim to identify fundamental parameters that significantly influence the performance of such a system.

Let us summarize the mathematical notations. Vectors, matrices, their transpose, conjugate, conjugate transpose and inverse are denoted as $\mathbf{a}, \mathbf{A},{ }^{T}, .^{*},{ }^{H}$ and.$^{-1}$ respectively. Absolute value and the second norm are denoted by $|$.$| and$ $\|.\| . \mathbf{I}_{b}$ represents the $b \times b$ identity matrix. $[\mathbf{A}]_{k, j}$ denotes the entry of the $k^{\text {th }}$ row, and the $j^{\text {th }}$ column of matrix $\mathbf{A}$.

The rest of the paper is organized as follows. In Section II, the difference between near- and far-field will be described in details. Afterward, further properties of ELAA are summarized in Section III. Then, the used model is introduced in Section IV. Subsequently, the results are presented in Section V. Finally, the conclusion is given in Section VI. 
Modeling the near-field of extremely large

aperture arrays in massive MIMO systems

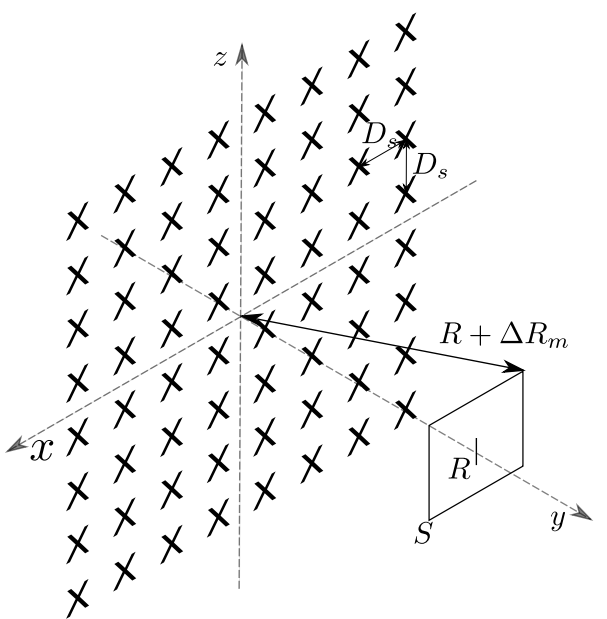

Figure 1. Scenario of 8 antenna element in the $x-z$ plane, $D_{s}=1 \mathrm{~m}$. The EM field is evaluated on the $S$ plane for different $R$.

\section{COMPARISON OF THE NEAR- AND FAR-FIELD}

Since our investigation principally assumes a radiating nearfield scenario, first let us point out the difference between radiating near-field and far-field. A well-defined boundary does not exist between the two regions, whereas there are distinct differences among them. For the sake of completeness, three different field regions can be identified surrounding an antenna: reactive near-field, radiating near-field (Fresnel region, also referred to as near-field), and far-field (Fraunhofer region) [8]. In the following parts, these regions will be discussed in detail.

For the sake of simplicity, the widely used criteria for each region are summarized first:

- reactive near-field: $0 \leq R<0.62 \sqrt{D^{3} / \lambda}$,

- radiating near-field (Fresnel region or simply near-field in the following): $0.62 \sqrt{D^{3} / \lambda} \leq R<R_{F}=2 D^{2} / \lambda$,

- far-field (Fraunhofer region): $R_{F} \leq R<\infty$,

where $R$ is the distance from the surface of the antenna, $D$ is the largest dimension of the aperture and $\lambda$ is the wavelength.

The closest one to the antenna is the reactive near-field, which is defined as "that portion of the near-field region immediately surrounding the antenna wherein the reactive field predominates" [8].

If $D$ is large compared to the wavelength, the Fresnel zone exists; otherwise, it does not. According to a definition in this region, "radiating field predominates, and the angular field distribution is dependent upon the distance from the antenna" [8]. Therefore, in the radiating near-field, spherical waves are utilized to describe the radiated electromagnetic field. In practical terms, even a small change in position might result in a significant change in the electromagnetic field.

In the far-field, "the field mainly has transverse component, and the angular field distribution is independent of the distance $(R)$ from the antenna" [8]. In the Fraunhofer region planewaves are utilized as a local approximation of the propagating field. Consequently, the EM field must not vary significantly on a surface element of a plane perpendicular to $R$ in the farfield. A rule of thumb for the size of this surface element is

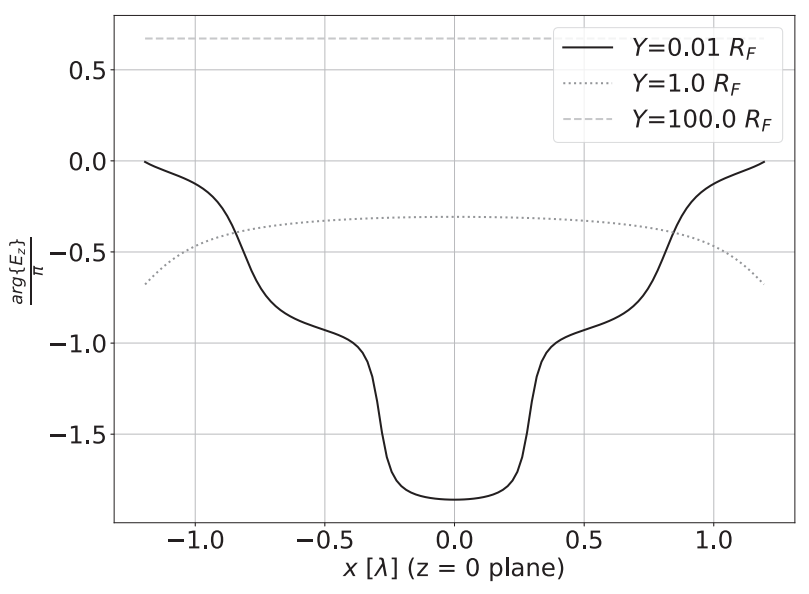

Figure 2. Illustrating the change in $\arg \left\{E_{z}\right\}$. In the near-field ( $Y=$ $\left.0.01 R_{F}\right)$, there is an abrupt change in the phase and $\arg \left\{E_{z}\right\} \in[-\pi, \pi]$. In the transition zone $\left(Y=R_{F}\right)$, there is rather continuous change in the phase and $\arg \left\{E_{z}\right\} \in[-0.918 \pi,-0.307 \pi]$. In the far-field $\left(Y=100 R_{F}\right)$, the change in phase can be neglected compared to the change in the transition zone.

$\Delta R_{m} \ll \lambda$. Where $\Delta R_{m}$ is the largest difference from $R$ in the distance from the antenna along with the surface element.

As an illustration, consider a scenario depicted in Figure 1. In this scenario, there are $8 \times 8$ antenna elements deployed on the $x-z$ plane in the nodes of an equidistant mesh, the spacing between the antenna elements is denoted by $D_{s}$, which is choosen to $1 \mathrm{~m}$. The size of the near-field is denoted in Table I. Half-wavelength dipoles (also referred to as dipoles) model each antenna element, for which electromagnetic field is known in closed form [8]. Free-space parameters have been used for the numerical evaluation, the phasor of the excitation of each dipole is $I_{0}=1 \mathrm{~A}$, with the frequency $f=2 \mathrm{GHz}$. For visualizing the difference between near-field and far-field, the complex amplitude of the $z$ component of the electric field $\left(E_{z}\right)$ is evaluated for a finite surface $(S)$. At the distance $R=$ $R_{F}$ the largest difference from $R$ in the distance along $S$ is $\Delta R_{m}=0.001 \lambda$. Since $\left|E_{z}\right|$ evaluated on $S$ does not vary significantly as $R$ increases, it is beneficial to focus on the phase of $E_{z}$ denoted as $\arg \left\{E_{z}\right\}$. Figure 2 depicts the phase for different $Y$ coordinates of the $S$ plane for a cross-section. Under near-field conditions $\left(Y \ll R_{F}\right)$, there is an abrupt change in $\arg \left\{E_{z}\right\}$, which indicates that the $S$ plane is in the Fresnel region. In the transition region $\left(Y=R_{F}\right)$, there is a small and smooth change in the phase. Lastly, in the far-field, it is of key importance that in the far-field the phase almost constant, which falls in line with our expectation.

As has been shown, there are differences between the radiating near-field and far-field of an aperture. If we are considering large antenna apertures, $R_{F}$ can easily be in the order of kilometers. Therefore users are located in the radiating near-field, which is a novel scenario compared to conventional MIMO systems.

\section{EXTREMELY LARGE APERTURE ARRAYS}

A conventional method to increase the number of antennas is to develop compact arrays that, for example, can be installed 
Table I

NUMERICAL EXAMPLES OF THE SIZE OF THE FRESNEL ZONE.

\begin{tabular}{c||c||c||c}
\hline$\lambda$ & $N_{\text {row }} \times N_{\text {col }}$ & $D_{s}$ & $R_{F}$ \\
\hline \hline $0.15 \mathrm{~m}$ & $8 \times 8$ & $1 \mathrm{~m}$ & $1307 \mathrm{~m}$ \\
\hline \hline $0.05 \mathrm{~m}$ & $20 \times 20$ & $3 \mathrm{~m}$ & $259.92 \mathrm{~km}$ \\
\hline \hline $0.1 \mathrm{~m}$ & $20 \times 20$ & $0.1 \mathrm{~m}$ & $144.4 \mathrm{~m}$ \\
\hline \hline $0.1 \mathrm{~m}$ & $20 \times 20$ & $1 \mathrm{~m}$ & $14.44 \mathrm{~km}$ \\
\hline \hline $0.1 \mathrm{~m}$ & $20 \times 20$ & $3 \mathrm{~m}$ & $129.96 \mathrm{~km}$ \\
\hline
\end{tabular}

on a rooftop. Such a massive MIMO array has become available on the market in recent years [9]. As going more massive, weight, and the wind load of the array are going to act as limiting factors. Extremely large aperture arrays are promising candidates to overcome these limitations. In such a scenario, the antenna elements are spread over a large area, fixed to existing physical structures, such as windows of a building. Thus, the aperture size of the antenna array is increased significantly.

Furthermore, ELAAs are beneficial from other aspects as well. If an antenna array has more than one main lobes, spatial aliasing appears. These main lobes, which appear because of spatial undersampling, are called grating lobes. Spatial undersampling happens if the antennas are more than a half wavelength apart. Despite introducing grating lobes, increasing the distance between the antenna elements further than half wavelength will result in better performance, as this has been shown by simulations [6], [5] and validated by measurements [10]. There are two main underlying effects behind the growing performance: finer spatial resolution and larger aperture near-field.

Spatial resolution is the measure of user separation capabilities. It depicts how closely placed users can be distinguished based on their channels. Interestingly, the spatial resolution depends on the size of the aperture, not the number of antennas [11]. As a demonstrative example, let us consider an equidistant antenna row. If the number of antennas gets doubled while the length of the array stays the same, the resulting spatial resolution would be identical. Spatial resolution is proportional to the width of the main lobe. Although the width of the main lobe is measured based on the far-field radiation pattern, it is also relevant in the near-field. Since as the size of the aperture increases, the width of the main lobe decreases, and users placed closer to each other can be differentiated.

The other main effect is that the radiating near-field of the aperture expands rapidly along with the size of the aperture. As can be concluded from a numerical example presented in Table I, in an ELAA scenario, the majority of the users fall into the radiating near-field. In the Fresnel region, there can be abrupt changes in both the amplitude and phase of the electromagnetic field. Constructive interferences in distinct points in space characterize the structure of the EM field. Nearfield effects will make user separation easier, this will result in better conditioned wireless channels, as it will be pointed in Section V-B. For example, users can be separated based on

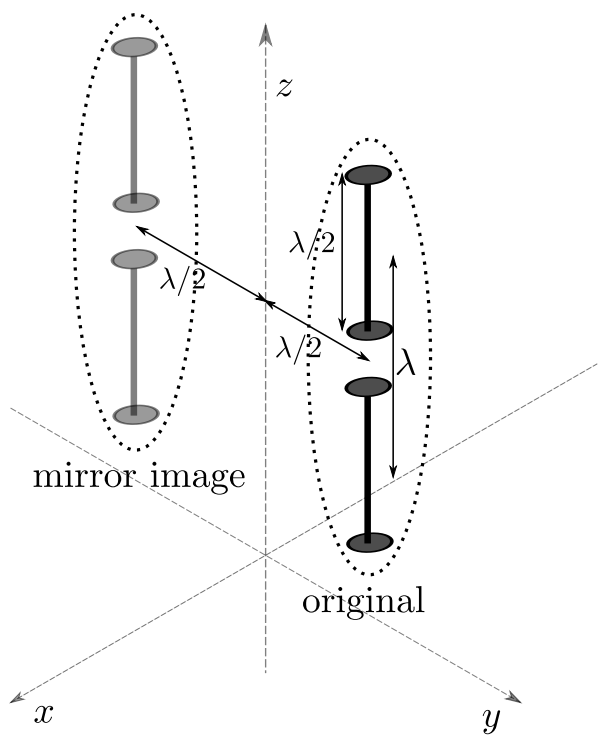

Figure 3. Constructing a simple directional antenna element from halfwavelength dipoles.

their channels, even if they are behind each other.

\section{MODEL DESCRIPTION}

In this paper, we are focusing on a single cell of a cellular network. The cell consists of an $M$-antenna base station and $K$ single-antenna mobile stations. In such a scenario, there is a radio channel between each BS and MS antenna. Flat fading channels are assumed, i.e., the channel gains are described by a single complex number [12]. Consequently, $\mathbf{h}_{k} \in \mathbb{C}^{M}, k=0,1, \ldots, K-1$ describes the channel of one terminal. By utilizing the channel vectors, the multi-user channel matrix can be built as

$$
\mathbf{H}=\left[\mathbf{h}_{0}, \mathbf{h}_{1}, \ldots, \mathbf{h}_{K-1}\right] \in \mathbb{C}^{M \times K} .
$$

Based on the models introduced in Section IV-A, IV-B and IV-C, the multi-user channel matrix corresponding to a particular scenario can be calculated. A well-accepted convention that the dimension of $\mathbf{H}$ is the number of receive antennas times the number of transmit antennas. Thus the above constructed $\mathbf{H}$ can also be referred to as the uplink (UL) channel matrix, whereas $\mathbf{H}^{T}$ is the downlink (DL) channel matrix. $\mathbf{H}$ is of crucial importance in the following parts. The multi-user channel matrix will be simulated in realistic scenarios and with different approximations.

\section{A. Propagation models}

Three different propagation models will be comapred. The first is a spherical-wave model, as in [5] and [7]

$$
h_{m, k}=\frac{1}{r_{m, k}} e^{-j \beta r_{m, k}} \in \mathbb{C},
$$

where $r_{m, k}$ is the distance between the $m^{\text {th }}$ MS and $k^{\text {th }}$ BS antenna and $\beta=\frac{2 \pi}{\lambda}$ is the wave number. The channel vector is constructed as

$$
\mathbf{h}_{k}=\left[h_{0, k}, h_{1, k}, h_{2, k}, \ldots, h_{M-1, k}\right]^{T} \in \mathbb{C}^{M} .
$$


Modeling the near-field of extremely large

aperture arrays in massive MIMO systems

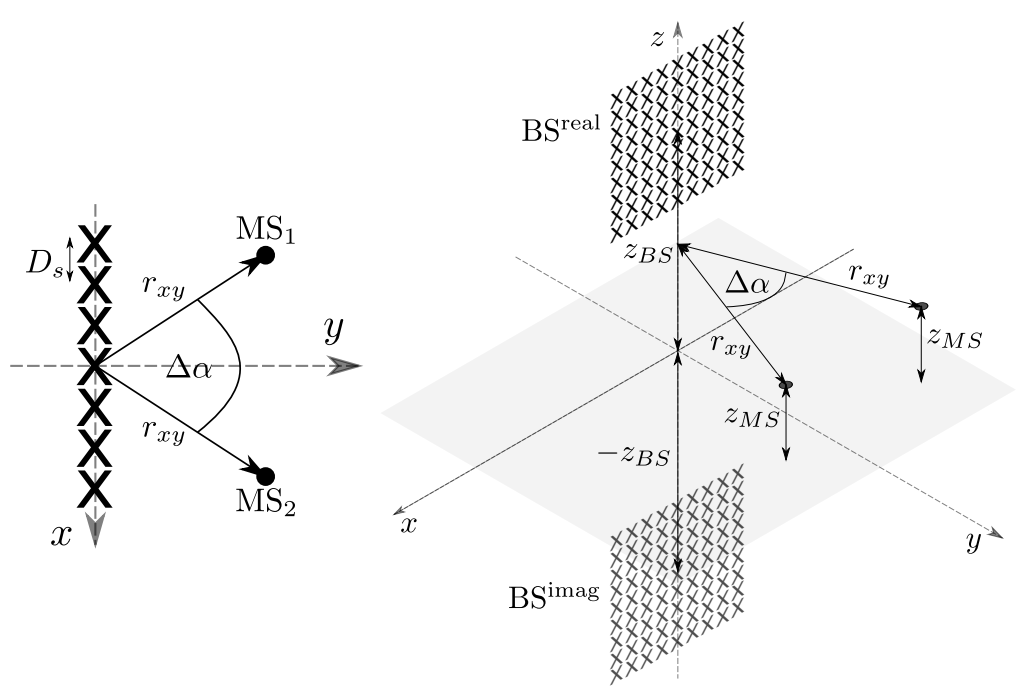

(a)

(b)

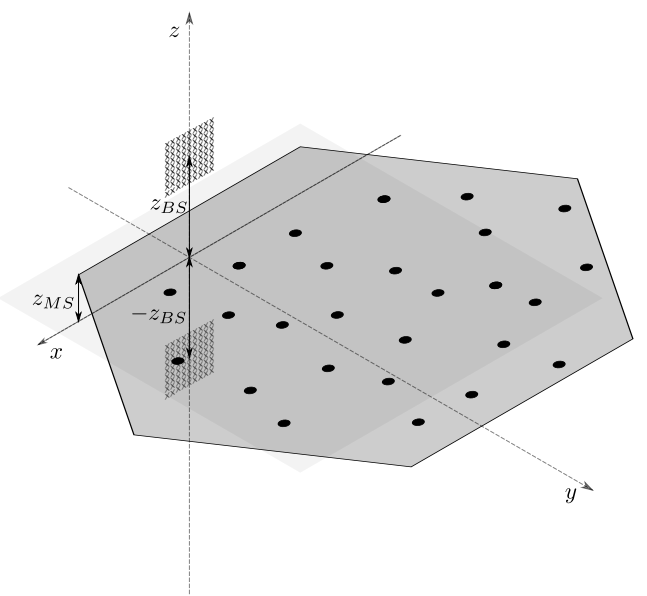

(c)

Figure 4. Different model scenarios. (a) depicts the one-dimensional case, whereas in (b), the two-dimensional case was drawn, with the mirror image antenna array utilized to model perfect reflection. In (c), uniformly distributed users are presented in a hexagonal cell.

Second, each antenna element is modeled by a halfwavelength dipole. At a given point in space, the field components of every dipole can be evaluated [8]. We assume that the dipoles are pointing toward the $z$ axis, just as the antennas of the terminals. Therefore, the received signal is proportional to the $z$ component of the electric field. As a result, the channel can be modeled as

$$
h_{m, k}=\frac{E_{z}^{m}\left(\vec{r}_{k}\right)}{I_{0}^{m}}
$$

where $E_{z}^{m} \in \mathbb{C}$ is a phasor which represents the $z$ component of the electric field created by the $m^{\text {th }}$ BS antenna, $\vec{r}_{k}$ is the position of the $k^{\text {th }}$ terminal. $I_{0}^{m} \in \mathbb{C}$ is a phasor - with the same frequency as $E_{z}^{m}$ - representing the excitation of the $m^{\text {th }}$ BS antenna. Let $I_{0}^{m}=1 \mathrm{~A}$ for arbitrary $m$. Again, the channel vector is constructed as (3).

Third, as the most realistic model, simple directional antenna elements were employed. A straightforward way to achieve directivity is to use two half-wavelength dipoles and a perfect reflector behind them. Both dipoles should point toward the $z$ axis. In spite of the fact that the distance between them is in the order of magnitude of wavelength, coupling effects will be neglected. This assumption will be supported by results that will be presented in Section V-B. The perfect reflector must be placed $\frac{\lambda}{2}$ from the dipoles to obtain the directivity. Subsequently, with an electrostatic analogy, the perfect reflector's effect can be modeled in terms of mirror image dipoles. Therefore, the resulting EM field will be identical if two other dipoles are placed behind the original ones at the distance of $\lambda$. As a result, each antenna is modeled by four half-wavelength dipoles. Since, the electromaganetic field of the dipoles are known analytically, $\mathbf{h}$ can be obtained similarly to (4) and (3) [8].

After the above-described models, a natural question might follow whether these models include the polarization of electromagnetic waves. The spherical-wave model neglects this ef- fect. However, if the field of the antenna elements is evaluated analytically, the polarization is also included. However, this only holds if the EM field is evaluated analytically. Therefore, from the evaluated electromagnetic field, a more accurate channel model can be derived.

\section{B. One- and two-dimensional arrays}

Two different model assumptions will be compared. The first approach is when only a one-dimensional array is considered. Thus, the mobile stations and the base station are in the same plane, as in Figure 4a.

In the second, novel approach, the antenna array is two dimensional, located in the $x-z$ plane, while users are in the $x-y$ plane. Mobile stations are assumed to be at a height of $z_{M S}$ above the ground. Furthermore, if we neglect the reflection from other buildings and obstacles, there is at least one significant reflection from the ground. Let us consider a perfect reflection. Thus, the utilization of a mirror image antenna array will result in an identical electromagnetic, as we did to obtain a directional pattern. Therefore, the channel model must be modified as follow

$$
h_{m, k}=h_{m, k}^{r e a l}+h_{m, k}^{i m a g},
$$

where $h_{m, k}^{r e a l}$ corresponds to the channel between the $m^{\text {th }}$ antenna of the MS and $k^{\text {th }}$ antenna of the real BS, whereas $h_{m, k}^{\text {imag }}$ represents the channel between the $m^{\text {th }}$ antenna of the MS and $k^{\text {th }}$ antenna of the mirror image BS. Figure $4 \mathrm{~b}$ depicts this above described assumption. Modeling the ground reflection by means of a mirror image antenna array also includes the effect of polarization change, since boundary conditions are fulfilled on the ground. 


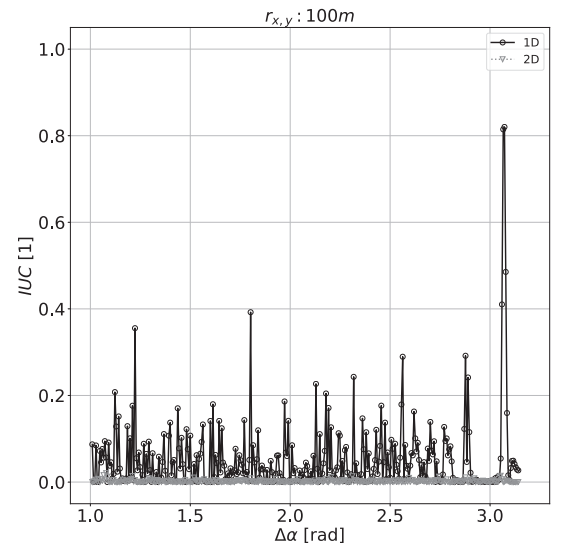

(a)

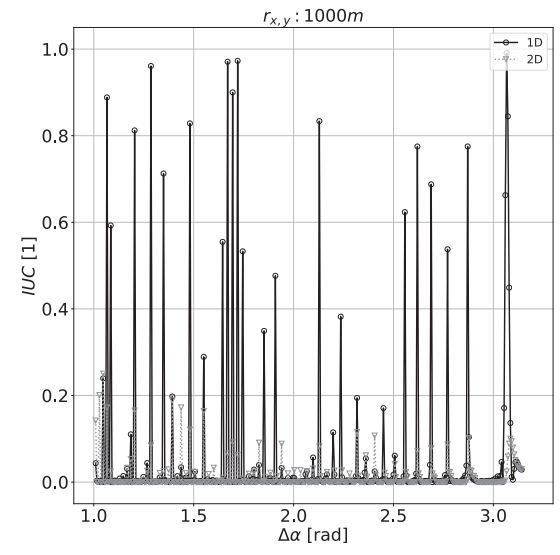

(b)

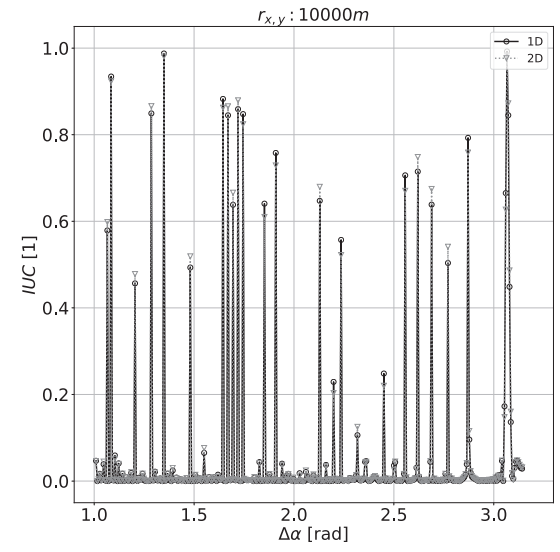

(c)

Figure 5. Comparing one- and two-dimensional models in terms of IUC $(\Delta \alpha)$.

\section{Array geometry}

Three different two-dimensional array geometries will be considered. The first one is an equidistant array, with parameters $N_{\text {row }} \times N_{\text {col }}$ and $D_{s}$.

Secondly, if we add random displacement to the $x$ and $z$ coordinates of each element of the equidistant case, a randomized array will be obtained. The displacements in both $x$ and $z$ directions are characterized by a normal distribution with standard deviation $\sigma_{D_{s}}$.

Lastly, we consider a scenario where the antenna elements are placed on a logarithmic spiral pattern. An analogy from acoustics inspired this scenario, where sensors are occasionally located on a spiral. The parameters of the spiral array are the distance of the closest and the farthest antenna element from the center of the array, the number of turns in the spiral and the number of antennas, denoted by $R_{\min }, R_{\max }$, $N_{\text {turn }}$ and $M$, respectively. In polar coordinates $(r, \phi)$, the equation of the logarithmic spiral is $r=R_{\min } e^{k \phi}$, where $k$ is a constant parameter, which calculated from $R_{\max }$ and $N_{\text {turn }}$. The antennas are positioned along with the pattern of the spiral, equal distance apart.

\section{Simulation RESUlts AND DisCUSSION}

A simulation framework has been developed in Python based on the models in Section IV. The metrics of the analysis will be introduced before the presentation of the results. Let us note here that a narrowband analysis is presented in this paper. However, the presented models hold in a wide frequency band. Investigating the behavior of the system for wideband signal excitation is left for further studies.

\section{A. Comparing one- and two-dimensional array models}

In order to compare the approaches mentioned in Section IV-B, the inter-user correlation will be evaluated between two users. The IUC is calculated as the inner product of the channel vectors of the two users

$$
\mathrm{IUC}=\frac{\left|\mathbf{h}_{1}^{H} \mathbf{h}_{2}\right|^{2}}{\left\|\mathbf{h}_{1}\right\|^{2}\left\|\mathbf{h}_{2}\right\|^{2}} .
$$

This quantity is a standard measure of the correlation of MIMO channels. The two users are located at the same distance from the BS ( denoted by $r_{x y}$ ), and the angle between them (denoted by $\Delta \alpha$ ) is varied, see Figure $4 \mathrm{a}$. As it is expected from (6), IUC $\in[0,1]$. Higher IUC indicates stronger interference between users. Therefore, lower IUC is desired, thus the inter-user interference is smaller, which results in higher spectral efficiency. Besides, the appearance of grating lobes $\left(D_{s} \geq \lambda / 2\right)$ or side lobes will introduce interuser correlation peaks.

A one-dimensional equidistant array with 20 antennas is simulated for the first modeling approach, only with the assumption of LoS propagation. For analyzing the second approach, a $20 \times 20$ equidistant array is utilized, and the ground reflection is accounted as well. In both cases spherical-wave propagation model is employed, $D_{s}$ is $3 \mathrm{~m}$ and $f=6 \mathrm{GHz}$. In the two-dimensional model, the center of the antenna array is at a height of $z_{M S}=40 \mathrm{~m}$ above ground and the terminals are located at a height of $z_{M S}=1.5 \mathrm{~m}$ above the ground. In such a scenario, the size of the near-field is $R_{F} \approx 260 \mathrm{~km}$, as shown in Table I. The two users were located at the distances $r_{x y}=\{100 \mathrm{~m}, 1 \mathrm{~km}, 10 \mathrm{~km}\}$. The results of the IUC analyses are shown in Figure 5. The employed antenna spacing $\left(D_{s}=3 \mathrm{~m}\right)$ gave rise to multiple peaks in the $\operatorname{IUC}(\Delta \alpha)$ graphs. As has been mentioned, this is due to the appearance of grating and side lobes. Closer to the array, the peaks correspond to smaller values, since the near-field effects mitigate the effect of grating lobes. As going farther from the array, peaks are becoming higher. Taking the reflection and real physical dimension into account results in lower IUC. Therefore if the simulation of a near-field scenario is required, the two-dimensional model is more accurate. Consequently, in the following simulations, the two-dimensional model will be utilized. On the contrary, the results of the two models converge to the same graph as the distance from the array increases, which is in line with our expectation. Thus, for farfield analysis, the simple model can be accurate enough. 
Modeling the near-field of extremely large

aperture arrays in massive MIMO systems

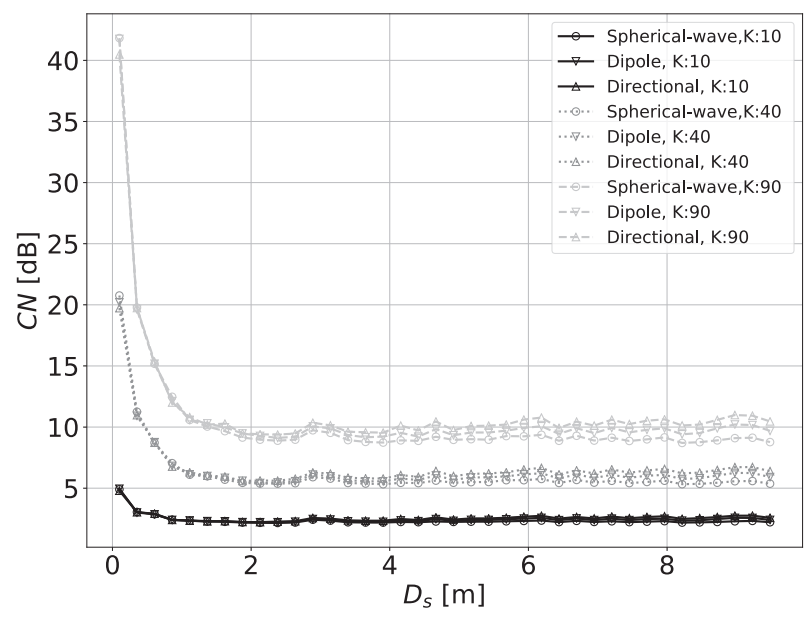

Figure 6. Condition number along with the element spacing of equidistant arrays for comparing propagation models.

\section{B. Comparison of antenna element models}

As has been described in Section IV-A, three different modeling approaches is considered: spherical-wave model, halfwavelength dipole, and a simple directional antenna element. The comparison is made in terms of the condition number of the multi-user channel matrix. The varying parameter is the spacing between the antenna elements $\left(D_{s}\right)$. An equidistant antenna array with $20 \times 20$ elements is simulated, $f=3 \mathrm{GHz}$, $z_{M S}=40 \mathrm{~m}$ and the users are located in a hexagonal cell right in front of the array, at a height of $z_{M S}=1.5 \mathrm{~m}$ above ground, as depicted in Figure 4c. The cell radius is $250 \mathrm{~m}$, and the users were uniformly distributed within the cell.

If the distance of the users from the BS differ significantly, power control will be required to provide the same service quality in the cell. There are multiple power control strategies [13]. In the following, an ideal power control technique will be used, thus the power of the received signal is identical for every user. Similarly to Normalization 1 in [14]

$$
\mathbf{H}^{\text {norm }}=\left[\frac{\mathbf{h}_{0}}{\left\|\mathbf{h}_{0}\right\|}, \frac{\mathbf{h}_{1}}{\left\|\mathbf{h}_{1}\right\|}, \ldots, \frac{\mathbf{h}_{K-1}}{\left\|\mathbf{h}_{K-1}\right\|}\right] \in \mathbb{C}^{M \times K} .
$$

s (7) sets the channel of each user to the same norm. The condition number is evaluated based on $\mathbf{H}^{\text {norm }}$ and defined as [5]

$$
\mathrm{CN}^{\mathrm{dB}}=20 \log _{10}\left(\frac{\sigma_{g}}{\sigma_{s}}\right),
$$

where $\sigma_{g}$ and $\sigma_{s}$ are the largest and smallest singular values of the normalized multi-user channel matrix $\left(\mathbf{H}^{\text {norm }}\right)$. The condition number strongly related to the orthogonality of the columns of the channel matrix. The evaluation of the inverse of $(\mathbf{H})$ is required for many massive MIMO transmission schemes. Positively, condition number also indicates the stability of the inversion [5]. In practical terms lower condition number indicates easier inversion and less correlated columns, for example, $\mathrm{CN}^{\mathrm{dB}}=0$ for an identity matrix, since its inverse is trivial and its columns are orthogonal.

The condition number has been evaluated in 1000 different realizations, with varying MS positions as described above.

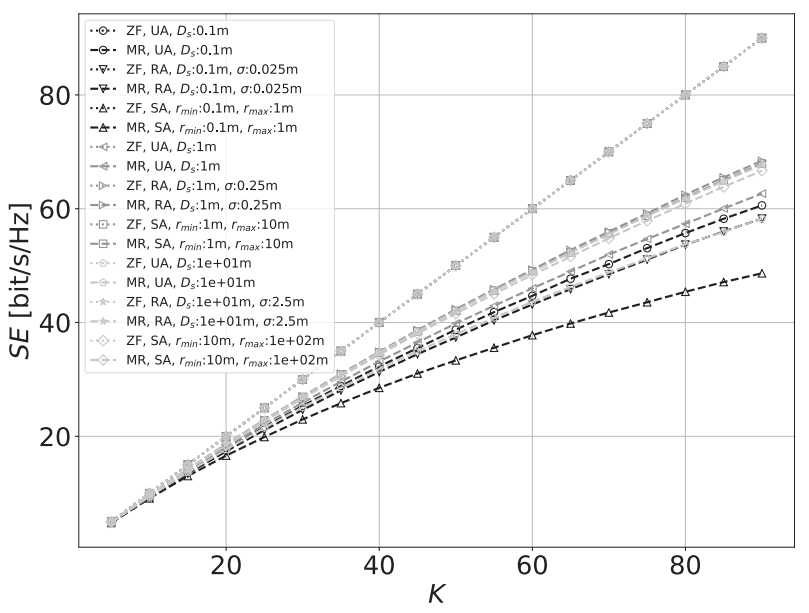

Figure 7. Cumulative spectral efficiency along with the number of users for different array geometries and precoding strategies. Dotted curves used for $\mathrm{ZF}$ precoding and dashed for MR, identical array settings are denoted with the same markers. The abbreviations EA, RA, SA stand for equidistant, randomized and spiral array, respectively.

In Figure 6 the average $\mathrm{CN}$ is plotted along $D_{s}$. The first observation is that the condition number shows a decreasing tendency as $D_{s}$ increases, independent of the used antenna model or the number of users. Therefore, increasing the size of the aperture is beneficial, as mentioned in Section III. Interesting observation, that in the first part $\left(D_{S} \in[\lambda, \approx 10 \lambda]\right)$ of the investigated $D_{s}$ range, the condition number decreases rapidly. In the second part $\left(D_{S} \in[\approx 10 \lambda, \approx 100 \lambda]\right)$, it becomes approximately constant, it even increases a little, for particular antenna models. Consequently, based on the scenario, an element spacing range can be specified, where the system can provide a well-conditioned channel.

One might question the periodic peaks in the $\mathrm{CN}\left(D_{s}\right)$ curves. This occurs because of the contribution of two effects. First, the reduction of the width of the main lobe and the correlation between the users are decreasing functions of $D_{s}$, whereas, grating lobes appear at discrete $D_{s}$ values, thus introducing peaks [5].

A relatively straightforward observation, that service quality will degrade if more users are transmitting simultaneously. Since for more terminals the mean of the $\mathrm{CN}^{\mathrm{dB}}\left(D_{s}\right)$ curves are clearly larger.

Finally, there is no significant difference between the used models in terms of condition number. Therefore, using the spherical-wave model is enough to acquire channel features. Besides, by using a more accurate model, a sightly higher condition number is obtained, which indicates that an upper boundary of the system performance will result from the spherical-wave model. Based on these results, including the polarization of the electromagnetic waves seems to have minor effects.

\section{Comparing different array geometries}

In Section IV-C two different array geometries have been defined. In the following, these two will be compared in 
terms of spectral efficiency. SE also gives an insight into the performance of the extremely large aperture array. The evaluation of the inter-user correlation and the condition number of the multi-user channel matrix are the same for uplink and downlink systems. On the contrary, spectral efficiency must be evaluated based on the direction of the data transmission. In the simulation, a downlink scenario is considered. In this case the received signal vector $\mathbf{y} \in \mathbb{C}^{K}$ is given by

$$
\mathbf{y}=\mathbf{H}^{T} \mathbf{W P x}+\mathbf{w},
$$

where $\mathbf{W} \in \mathbb{C}^{M \times K}$ is the precoding matrix, $\mathbf{P} \in \mathbb{C}^{K \times K}$ is a diagonal matrix capturing the effects of the power control, $\mathbf{x} \in \mathbb{C}^{K}$ is the transmit signal vector and $\mathbf{w} \in \mathbb{C}^{K}$ represents the additive white Gaussian noise, $\mathbf{w} \sim \mathcal{C N}\left(0, \sigma_{D L}^{2} \mathbf{I}_{K}\right)$. For the transmit signal vector $\mathbb{E}\left\{\mathbf{x x}^{H}\right\}=\mathbf{I}_{K}$ holds. Each column $\mathbf{w}_{k} \in \mathbb{C}^{M}$ of $\mathbf{W}$ corresponds to the coefficients which are used to precode the data $x_{k}$ dedicated to the $k^{\text {th }}$ MS.

Similarly to (7), the power control can be expressed by a diagonal matrix, constructed as

$$
\mathbf{P}=\operatorname{diag}\left[\frac{\rho_{0}}{\left\|\mathbf{h}_{0}\right\|}, \frac{\rho_{1}}{\left\|\mathbf{h}_{1}\right\|}, \ldots, \frac{\rho_{K-1}}{\left\|\mathbf{h}_{K-1}\right\|}\right] \in \mathbb{C}^{K \times K},
$$

where the square of $\rho_{k}, k=0,1, \ldots, K-1$ represents the target transmit power for user $k$. For the simulation, we consider $\rho_{k}=\rho_{D L}, \forall k$. Consequently, $\mathbf{P}=\rho_{D L} \mathbf{I}_{K}$. Two different precoding strategies are considered. The first one is the maximum ratio (MR) processing, in this case

$$
\mathbf{W}_{M R}=\left(\mathbf{H}^{\text {norm }}\right)^{*},
$$

where $\mathbf{H}^{\text {norm }}$ is obtained as (7). The underlying idea of the MR processing is to amplify the signal of interest maximally and neglect the effect of interference [13]. The second is zeroforcing $(\mathrm{ZF})$ processing which aims to mitigate interference among users, by the utilization of (7)

$$
\mathbf{W}_{Z F}=\left(\mathbf{H}^{\mathrm{norm}}\right)^{*}\left(\left(\mathbf{H}^{\mathrm{norm}}\right)^{T}\left(\mathbf{H}^{\mathrm{norm}}\right)^{*}\right)^{-1} .
$$

In (12) the requirement of the inversion is fulfilled if the user channels are orthogonal, which holds if the base station is equipped with a sufficient number of antennas [15]. (11) and (12) are almost identical to (3.57) and (3.49) in [13], the multiplicative constants is removed because of the used power control strategy.

It can be concluded from (9) that in order to transmit data $x_{k}$ which is dedicated to the $k^{\text {th }}$ MS, $x_{k}$ is multiplied by $[\mathbf{W}]_{m, k}$ before it is being transmitted at the $m^{\text {th }}$ BS antenna. As a consequence, in a distributed system with one antenna, it is enough to store a single row of $\mathbf{W}$. The maximum ratio precoding has a considerable advantage that it can be deployed in a distributed system because each row of $\mathbf{W}_{M R}$ can be obtained by knowing the corresponding row of $\mathbf{H}^{\text {norm }}$. In contrast, ZF processing requires centralized control because of the calculation of $\mathbf{W}_{Z F}$. On the other hand, zero-forcing will deliver higher SE than maximum ratio processing.

The evaluation of the downlink spectral efficiency simplifies owing to the simulated $\mathbf{H}$, which provides a perfect channel state information. Therefore, the expectations in (4.26) from
[11] simplifies and the signal-to-interference-noise can be expressed as

$$
\operatorname{SINR}_{k}=\frac{\left|\left[\mathbf{H}^{T} \mathbf{W} \rho_{D L}\right]_{k, k}\right|^{2}}{\sum_{j=0, j \neq k}^{K-1}\left|\left[\mathbf{H}^{T} \mathbf{W} \rho_{D L}\right]_{k, k}\right|^{2}+\sigma_{D L}^{2}},
$$

where $\mathbf{P}=\rho_{D L} \mathbf{I}_{K}$ and continuous downlink transmission is assumed. Thus, the lower bound of the sum ergodic downlink spectral efficiency is

$$
\mathrm{SE}=\sum_{k=0}^{K-1} \log _{2}\left(1+\mathrm{SINR}_{k}\right) .
$$

In the simulation, $\mathbf{H}$ is known explicitly based on a particular realization. Therefore in (13) and (14) all the variables can be considered as deterministic. In order to simulate the random behavior of the users, spectral efficiency is calculated in 1000 different scenarios, with equally distributed random user positions within the cell. The average SE is visible in Figure 7. The parameters of the simulation are considered as for the evaluation of condition number, described in Section V-B. The only differences are the array geometry and postprocessing, since spectral efficiency is calculated, with $\rho_{D L}=\sigma_{D L}=1$. The changing parameters $\sigma_{D_{s}}, R_{\min }, R_{\max }$ are denoted on the obtained cruves, whereas $N_{\text {turn }}=4$ is constant.

The first observation is that the ZF precoding significantly outperforms the MR precoding, independent of array geometry and aperture size. Therefore, the core concept of $\mathrm{ZF}$ precoding is realized here, since it has successfully suppressed all the interference between the users. Thus the curves corresponding to $\mathrm{ZF}$ precoding overlap. Also, $\mathrm{ZF}$ is more complex to implement because of its centralized fashion. Therefore, the comparison based on MR precoding is more relevant from practical perspectives.

It is important to note that in Figure 7 the cumulative spectral efficiency is plotted, thus in case of the MR precoding, spectral efficiency of a single user is a decreasing function of $K$. Therefore, the size of the system is limited by the target spectral efficiency of a single user. If higher SE is required for a single user, the number of BS antennas must be increased. However, it is essential to keep it at least an order of magnitude higher than the number of concurrently transmitting terminals.

As it was expected, increasing the size of the aperture resulted in a higher spectral efficiency, if we are considering MR precoding. This increment is significant if $D_{s}=0.1 \mathrm{~m}$ and $D_{s}=1 \mathrm{~m}$ are compared. However, the difference between the $D_{s}=1 \mathrm{~m}$ and $D_{s}=10 \mathrm{~m}$ is negligible. Furthermore, in case of the spiral array, $r_{\text {min }}=10 \mathrm{~m}$ array is outperformed by the $r_{\text {min }}=1 \mathrm{~m}$ array. The results corresponds with the $\mathrm{CN}\left(D_{s}\right)$ curves in Figure 6, since the condition number does not vary significantly for $D_{s}>\approx 1 \mathrm{~m}$.

Finally, if large apertures are considered, there is no significant difference between the different array geometries. Even though the random and equidistant arrays outperform the spiral array for small apertures, as increasing the distance between the antennas, this difference diminishes. Consequently, the positions of the array elements of a realized ELAA can almost be arbitrary, which is beneficial from a practical perspective. 
Modeling the near-field of extremely large

aperture arrays in massive MIMO systems

\section{CONCLUSION}

In the presented work, we analyzed the effect of modeling accuracy, aperture geometry and different antenna implementations in typical near-field ELAA scenarios. We demonstrated that simple line-of-sight propagation models overestimate inter-user correlation, whereas a more accurate model that considers ground reflections yields improved user separation capability, thus emphasizing the need for more detailed, physically motivated channel modeling approaches. Furthermore, it has been shown that the performance of the system only weakly depends on the actual implementation of the antenna elements. The condition number of the multi-user channel matrix will show similar statistics irrespective of the accuracy of the field strength calculation (simple ray-based vs. analytical calculation of the electromagnetic field). Finally, we demonstrated that increasing the size of the aperture, at least to some extent, while keeping the number of antennas identical, will yield higher spectral efficiency. On the other hand, the results are relatively independent on the actual array geometry. Therefore, antenna elements can be placed almost arbitrarily, which is beneficial from a practical implementation perspective. In conclusion, the size of the aperture can be identified as a key design parameter, with spectral efficiency being the design objective.

\section{ACKNOWLEDGMENT}

The research peresented in this paper was supported by the EFOP-3.6.2-16-2017-00013 project.

\section{REFERENCES}

[1] T. L. Marzetta, "Massive MIMO: an introduction," Bell Labs Technical Journal, vol. 20, pp. 11-22, 2015, DOI: 10.15325/BLTJ.2015.2407793.

[2] E. Björnson, J. Hoydis, and L. Sanguinetti, "Massive MIMO has unlimited capacity," IEEE Transactions on Wireless Communications, vol. 17, no. 1, pp. 574-590, 2017, Dor: 10.1109/TWC.2017.2768423.

[3] Björnson, Emil and Sanguinetti, Luca and Wymeersch, Henk and Hoydis, Jakob and Marzetta, Thomas L, "Massive MIMO is a realityWhat is next?: Five promising research directions for antenna arrays," Digital Signal Processing, 2019, DoI: 10.1016/j.dsp.2019.06.007.

[4] A. Amiri, M. Angjelichinoski, E. De Carvalho, and R. W. Heath, "Extremely large aperture massive MIMO: Low complexity receiver architectures," in 2018 IEEE Globecom Workshops. 9-13 Dec. 2018, Abu Dhabi, United Arab Emirates: IEEE, 2018, pp. 1-6, DoI: $10.1109 /$ GLOCOMW.2018.8644126

[5] D. Pinchera, M. D. Migliore, F. Schettino, and G. Panariello, "Antenna arrays for line-of-sight massive MIMO: Half wavelength is not enough," Electronics, vol. 6, no. 3, p. 57, 2017, DoI: $10.3390 /$ electronics6030057.

[6] S. Pratschner, E. Zöchmann, H. Groll, S. Caban, S. Schwarz, and M. Rupp, "Does a large array aperture pay off in line-of-sight massive MIMO?" in 2019 IEEE 20th International Workshop on Signal Processing Advances in Wireless Communications. 2-5 July 2019, Cannes, France: IEEE, 2019, pp. 1-5, DoI: 10.1109 /SPAWC. 2019.8815590
[7] Z. Zhou, X. Gao, J. Fang, and Z. Chen, "Spherical wave channel and analysis for large linear array in LoS conditions," in 2015 IEEE Globecom Workshops. 6-10 Dec. 2015, San Diego, CA: IEEE, 2015, pp. 1-6, Dor: 10.1109/GLOCOMW.2015.7414041.

[8] C. A. Balanis, Antenna theory: analysis and design, 3rd ed. John Wiley \& Sons, 2005, ISBN: 9780471667827.

[9] E. Björnson, "A look at an LTE-TDD massive MIMO product," acessed: 2020-10-05. [Online]. Available: http://ma-mimo.ellintech. se/2018/08/27/a-look-at-an-lte-tdd-massive-mimo-product/

[10] À. O. Martínez, E. DeCarvalho, and J. Ø. Nielsen, "Towards very large aperture massive MIMO: A measurement based study," in 2014 IEEE Globecom Workshops. 8-12 Dec. 2014, Austin, TX: IEEE, 2014, pp. 281-286, DoI: 10.1109/GLOCOMW.2014.7063445.

[11] E. Björnson, J. Hoydis, and L. Sanguinetti, "Massive MIMO networks: Spectral, energy, and hardware efficiency," Foundations and Trends in Signal Processing, vol. 11, no. 3-4, pp. 154-655, 2017, DOI: $10.1561 / 2000000093$

[12] B. Sklar, "Rayleigh fading channels in mobile digital communication systems. i. characterization," IEEE Communications magazine, vol. 35, no. 7, pp. 90-100, 1997, DoI: $10.1109 / 35.601747$.

[13] T. L. Marzetta, Fundamentals of massive MIMO, 1st ed. Cambridge University Press, 2016, Dor: 10.1017/CBO9781316799895.

[14] X. Gao, O. Edfors, F. Rusek, and F. Tufvesson, "Massive MIMO performance evaluation based on measured propagation data," IEEE Transactions on Wireless Communications, vol. 14, no. 7, pp. 3899-3911, 2015, DoI: 10.1109/TWC.2015.2414413.

[15] H. Prabhu, J. Rodrigues, O. Edfors, and F. Rusek, "Approximative matrix inverse computations for very-large mimo and applications to linear precoding systems," in 2013 IEEE Wireless Communications and Networking Conference. 7-10 April 2013, Shanghai: IEEE, 2013, pp. 2710-2715, Dor: $10.1109 / \mathrm{WCNC} .2013 .6554990$.

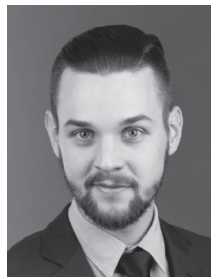

Botond Tamás Csathó is an MSc student at the Budapest University of Technology and Economics specialized in Wireless Networks and Applications. He received his $\mathrm{BSc}$ in 2019 at the same institute. His research interest focuses on massive MIMO networks, channel modeling and channel estimation. $\mathrm{He}$ is the member of the BME Balatonfüred Student Research Group.

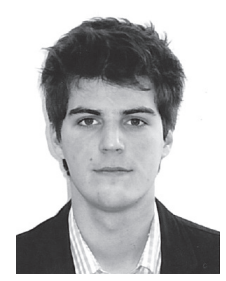

Bálint Péter Horváth received his M.Sc. (2013) and Ph.D. (2018) degrees in Electrical Engineering from the Budapest University of Technology and Economics where he is currently a senior lecturer at the Department of Broadband Infocommunications and Electromagnetic Theory. His research interests include signal processing in communications systems, software defined radio and computational model validation of portable wireless devices.

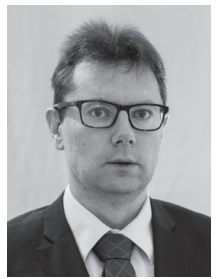

Péter Horváth obtained his Diploma degree at Budapest University of Technology and Economics (BME) and at Technical University of Karlsruhe, Germany. He received his PhD at BME in 2010, then he was a postdoctoral researcher at Vanderbilt University, USA. He is currently an associate professor in the Department of Broadband Infocommunications and Electromagnetic Theory at BME. His research interests include mobile wireless communications, cognitive radio and dynamic spectrum access technologies, channel modeling in multiple-antenna systems and signal design for satellite communications. 